\title{
Reachability relations and the structure of transitive digraphs
}

\author{
Norbert Seifter \\ Montanuniversität Leoben, Leoben, Austria \\ seifter@unileoben.ac.at \\ Vladimir I. Trofimov* \\ Russian Academy of Sciences, Ekaterinburg, Russia \\ trofimov@imm.uran.ru
}

Submitted: Dec 19, 2007; Accepted: Feb 17, 2009; Published: Feb 27, 2009

Mathematics Subject Classification: 05C25, 05C20

\begin{abstract}
In this paper we investigate reachability relations on the vertices of digraphs. If $W$ is a walk in a digraph $D$, then the height of $W$ is equal to the number of edges traversed in the direction coinciding with their orientation, minus the number of edges traversed opposite to their orientation. Two vertices $u, v \in V(D)$ are $R_{a, b^{-}}$ related if there exists a walk of height 0 between $u$ and $v$ such that the height of every subwalk of $W$, starting at $u$, is contained in the interval $[a, b]$, where $a$ ia a non-positive integer or $a=-\infty$ and $b$ is a non-negative integer or $b=\infty$. Of course the relations $R_{a, b}$ are equivalence relations on $V(D)$. Factorising digraphs by $R_{a, \infty}$ and $R_{-\infty, b}$, respectively, we can only obtain a few different digraphs. Depending upon these factor graphs with respect to $R_{-\infty, b}$ and $R_{a, \infty}$ it is possible to define five different "basic relation-properties" for $R_{-\infty, b}$ and $R_{a, \infty}$, respectively.

Besides proving general properties of the relations $R_{a, b}$, we investigate the question which of the "basic relation-properties" with respect to $R_{-\infty, b}$ and $R_{a, \infty}$ can occur simultaneously in locally finite connected transitive digraphs. Furthermore we investigate these properties for some particular subclasses of locally finite connected transitive digraphs such as Cayley digraphs, digraphs with one, with two or with infinitely many ends, digraphs containing or not containing certain directed subtrees, and highly arc transitive digraphs.
\end{abstract}

\footnotetext{
*Supported in part by the Russian Foundation for Basic Research (grant 06-01-00378). The work was done in part during the visit of Montanuniversität Leoben, Leoben, Austria in May, 2006.
} 


\section{Introduction}

In this paper we consider digraphs (i.e. directed graphs) which contain neither loops nor multiple edges. Thus, if $D$ is a digraph, then $E(D) \subseteq(V(D) \times V(D)) \backslash \operatorname{diag}(V(D) \times V(D))$ where $V(D)$ is the vertex set and $E(D)$ is the edge set of $D$. If $(u, v) \in E(D)$, then $u$ is called the initial vertex and $v$ is called the terminal vertex of the edge $(u, v)$. For $v \in V(D)$, $\operatorname{deg}_{D}^{-}(v):=|\{u:(u, v) \in E(D)\}|$ is the in-degree of $v, \operatorname{deg}_{D}^{+}(v):=|\{u:(v, u) \in E(D)\}|$ is the out-degree of $v$. A digraph $D$ is locally finite if $\operatorname{both}_{,} \operatorname{deg}_{D}^{-}(v)$ and $\operatorname{deg}_{D}^{+}(v)$, are finite for every $v \in V(D)$. For a digraph $D$ we denote the underlying undirected graph of $D$ by $\bar{D}$. So $\bar{D}$ is the graph with vertex set $V(\bar{D})=V(D)$ and edge set $E(\bar{D})=\{\{u, v\}$ : $(u, v) \in E(D)$ or $(v, u) \in E(D)\}$. We call a digraph $D$ connected if $\bar{D}$ is connected.

In this paper we frequently consider quotient digraphs with respect to partitions of their vertex sets. If $D$ is digraph and $\tau$ a partition of $V(D)$, then the vertex set of the quotient graph $D / \tau$ is given by the sets of $\tau$, and, for vertices $u^{\tau}$ and $v^{\tau}$ of $D / \tau,\left(u^{\tau}, v^{\tau}\right) \in E(D / \tau)$ if and only if $u^{\tau} \neq v^{\tau}$ and there exist $u_{1} \in u^{\tau}, v_{1} \in v^{\tau}$ such that $\left(u_{1}, v_{1}\right) \in E(D)$. If $R$ is the equivalence relation on $V(D)$ determined by $\tau$, we also denote the digraph $D / \tau$ by $D / R$.

For a digraph $D, \operatorname{Aut}(D)$ denotes the automorphism group of $D$. If $G \leq \operatorname{Aut}(D)$ and $\tau$ is a partition on $V(D)$ determined by a $G$-invariant equivalence relation $R$, then $G$ induces a group of automorphisms of $D / \tau$ denoted by $G^{\tau}$ or $G^{R}$. If $\operatorname{Aut}(D)$ acts transitively on $V(D)$ then we call $D$ a transitive digraph and clearly all vertices of a transitive digraph $D$ have the same in-degree $\operatorname{deg}^{-}(D)$ and the same out-degree $\operatorname{deg}^{+}(D)$.

A walk $W$ in a digraph $D$ is a sequence $\left(v_{0}, e_{1}^{\epsilon_{1}}, v_{1}, \ldots, v_{n-1}, e_{n}^{\epsilon_{n}}, v_{n}\right)$, where $n$ is a non-negative integer, $v_{0}, \ldots, v_{n} \in V(D), e_{1}, \ldots, e_{n} \in E(D)$ (if $n>0$ ) and $\epsilon_{1}, \ldots, \epsilon_{n} \in$ $\{-1,1\}$, such that, for each $1 \leq i \leq n$, either $\epsilon_{i}=1$ and $e_{i}=\left(v_{i-1}, v_{i}\right)$ or $\epsilon_{i}=-1$ and $e_{i}=\left(v_{i}, v_{i-1}\right)$. The vertices $v_{0}$ and $v_{n}$ are called the initial and terminal vertex of the walk $W$, respectively. The parameter $n$ is called the length $l(W)$ of $W$. For a walk $W=\left(v_{0}, e_{1}^{\epsilon_{1}}, v_{1}, \ldots, v_{n-1}, e_{n}^{\epsilon_{n}}, v_{n}\right)$ we define the height ht $(W)$ of $W$ as $h t(W)=\sum_{i=1}^{i=n} \epsilon_{i}$. If $n=0$, we set ht $(W)=0$.

If $W=\left(v_{0}, e_{1}^{\epsilon_{1}}, v_{1}, \ldots, v_{n-1}, e_{n}^{\epsilon_{n}}, v_{n}\right)$ is a walk in a digraph $D$, then, for any pair $i, j$, $0 \leq i \leq j \leq n$, the walk $\left(v_{i}, e_{i+1}^{\varepsilon_{i+1}}, \ldots, e_{j}^{\varepsilon_{j}}, v_{j}\right)$ is called the $(i, j)$-subwalk of $W$ and denoted by $W_{i, j}$.

In the sequel we say that $a \in \mathbf{Z}_{\leq \mathbf{0}} \cup\{-\infty\}$ if $a$ is a non-positive integer or $a=-\infty$ and $b \in \mathbf{Z}_{\geq \mathbf{0}} \cup\{\infty\}$ if $b$ is a non-negative integer or $b=\infty$. Let $D$ be a digraph. Furthermore, let $a \in \mathbf{Z}_{\leq \mathbf{0}} \cup\{-\infty\}$ and let $b \in \mathbf{Z}_{\geq \mathbf{0}} \cup\{\infty\}$. Denote by $\mathcal{R}_{a, b}^{D}$ the set of walks $W$ of $D$ with $\operatorname{ht}(W)=0$ and $a \leq \mathrm{ht}\left(W_{0, j}\right) \leq b$ for each $j, 0 \leq j \leq l(W)$. The $(a, b)$-reachability relation $R_{a, b}^{D}$ (or simply $R_{a, b}$ ) on $V(D)$ is defined in the following way: $u R_{a, b}^{D} v$ if there exists a walk $W$ in $\mathcal{R}_{a, b}^{D}$ with initial vertex $u$ and terminal vertex $v$.

It is easy to see that $R_{a, b}$ is an $\operatorname{Aut}(D)$-invariant equivalence relation on $V(D)$. The equivalence class of a vertex $v \in V(D)$ with respect to $R_{a, b}$ is denoted by $R_{a, b}(v)$. If $a^{\prime} \in \mathbf{Z}_{\leq \mathbf{0}} \cup\{-\infty\}$ such that $a^{\prime} \leq a$ and $b^{\prime} \in \mathbf{Z}_{\geq \mathbf{0}} \cup\{\infty\}$ such that $b^{\prime} \geq b$, then obviously $R_{a, b} \subseteq R_{a^{\prime}, b^{\prime}}$. Of course, $u R_{0,0} v$ if and only if $u=v$. (Note that the definition of $R_{a, b}$ can be naturally generalized to digraphs with loops, but, for transitive connected digraphs 
with loops, any such relation with $a<0$ or $b>0$ is universal.) Furthermore, it is easy to see that $R_{a, b}$ is the equivalence relation generated by $R_{a, 0}$ and $R_{0, b}$ (i.e. the smallest equivalence relation on $V(D)$ containing $R_{a, 0}$ as well as $R_{0, b}$ ). Thus, to consider $R_{a, b}$, it is sometimes sufficient to consider $R_{a}:=R_{a, 0}$ and $R_{b}:=R_{0, b}$.

We emphasize that - motivated by a problem posed in [1] - the reachability relations $R_{a}$ and $R_{b}$ were introduced in [8]. Moreover, it was already shown in [8] that there is a strong connection between properties of $R_{a}$ and $R_{b}$ and various other properties of digraphs. In this paper we study a more general concept of relations, namely the above defined $R_{a, b}$. We prove basic properties of relations $R_{a, b}$ which are in some cases generalizations of results shown in [8]. Besides that we are mainly interested in structural and algebraic properties of transitive digraphs when $a \rightarrow-\infty$ and $b \rightarrow+\infty$ for $R_{a}$ and $R_{b}$, respectively.

A connected digraph $D$ is a cycle if $|V(D)|$ is finite and either $|V(D)|=1$ (in which case $E(D)=\emptyset$ and $D$ is a trivial cycle) or every vertex of $D$ has in-degree 1 and out-degree 1. A digraph $D$ is a directed tree if $\bar{D}$ is a tree, and is a regular directed tree if $\bar{D}$ is a tree and all vertices of $D$ have the same in-degree and the same out-degree. A regular directed tree $D$ is called a chain if $\operatorname{deg}^{-}(D)=\operatorname{deg}^{+}(D)=1$. It is easy to see that any connected transitive digraph $D$ with $\operatorname{deg}^{-}(D) \leq 1$ is either a cycle or a chain or a regular directed tree with in-degree 1 and out-degree $>1$. Analogously, any connected transitive digraph $D$ with $\operatorname{deg}^{+}(D) \leq 1$ is either a cycle or a chain or a regular directed tree with in-degree $>1$ and out-degree 1 .

It can be proven (see Corollary 2.6 below) that, for any digraph $D$ and for any $a \in$ $\mathbf{Z}_{\leq \mathbf{0}} \cup\{-\infty\}$ and $b \in \mathbf{Z}_{\geq \mathbf{0}} \cup\{+\infty\}$, the in-degree of every vertex of $D / R_{a,+\infty}$ is $\leq 1$ and the out-degree of every vertex of $D / R_{-\infty, b}$ is $\leq 1$. Thus (see Corollary 2.7 below), for any connected transitive digraph $D$, the (connected transitive) digraph $D / R_{a,+\infty}$ is either a cycle or a chain or a regular directed tree with in-degree 1 and out-degree $>1$. Moreover (see Proposition 2.1 below), either $D / R_{a,+\infty}$ is a cycle for all $a \in \mathbf{Z}_{\leq \mathbf{0}} \cup\{-\infty\}$, or $D / R_{a,+\infty}$ is a chain for all $a \in \mathbf{Z}_{\leq \mathbf{0}} \cup\{-\infty\}$, or $D / R_{a,+\infty}$ is a regular directed tree with in-degree 1 and out-degree $>1$ for all $a \in \mathbf{Z}_{\leq \mathbf{0}}$. Analogously (see Corollary 2.7 below), for any connected transitive digraph $D$, the (connected transitive) digraph $D / R_{-\infty, b}$ is either a cycle or a chain or a regular directed tree with in-degree $>1$ and out-degree 1 . Moreover (see Proposition 2.1 below), either $D / R_{-\infty, b}$ is a cycle for all $b \in \mathbf{Z}_{\geq \mathbf{0}} \cup\{+\infty\}$, or $D / R_{-\infty, b}$ is a chain for all $b \in \mathbf{Z}_{\geq \mathbf{0}} \cup\{+\infty\}$, or $D / R_{-\infty}, b$ is a regular directed tree with in-degree $>1$ and out-degree 1 for all $b \in \mathbf{Z}_{\geq \mathbf{0}}$. In particular, we get that, for any connected transitive digraph $D$, the (connected transitive) digraph $D / R_{-\infty,+\infty}$ is either a cycle or a chain.

Furthermore, if $D$ is a digraph and $a \in \mathbf{Z}_{\leq \mathbf{0}} \cup\{-\infty\}$, then either $R_{a, k+1}=R_{a, k}$ for some positive integer $k$ and $R_{a,+\infty}=R_{a, k}$, or $R_{a, k+1} \neq R_{a, k}$ and $R_{a,+\infty} \neq R_{a, k}$ for any positive integer $k$ (see Proposition 2.5 below). It follows from Corollary 2.4 (see below) that, for a digraph $D$, the property to satisfy $R_{a,+\infty}=R_{a, k}$ for some positive integer $k$ holds either for all or for none of non-positive integers $a$, i.e. this property of $D$ is independent of the choice of non-positive integer $a$. Thus we can formulate this property of $D$ as $R_{+\infty}=R_{k}$ for some positive integer $k$. Analogously, if $D$ is a digraph and $b \in \mathbf{Z}_{\geq \mathbf{0}} \cup\{+\infty\}$, then either $R_{-k-1, b}=R_{-k, b}$ for some positive integer $k$ and $R_{-\infty, b}=R_{-k, b}$, or $R_{-k-1, b} \neq R_{-k, b}$ 
and $R_{-\infty, b} \neq R_{-k, b}$ for any positive integer $k$ (see Proposition 2.5 below). It follows from Corollary 2.4 (see below) that, for a digraph $D$, the property to satisfy $R_{-\infty, b}=R_{-k, b}$ for some positive integer $k$ holds either for all or for none of non-negative integers $b$, i.e. this property of $D$ is independent of the choice of non-negative integer $b$. Thus we can formulate this property of $D$ as $R_{-\infty}=R_{-k}$ for some positive integer $k$.

It can be shown (see Corollary 2.12 and Proposition 2.10 below) that $R_{-\infty}=R_{-k}$ for some positive integer $k$ in the case $D / R_{-\infty}$ is finite, and, analogously, $R_{+\infty}=R_{k}$ for some positive integer $k$ in the case $D / R_{+\infty}$ is finite. Thus, for any connected transitive digraph $D$ and any $\epsilon \in\{-,+\}$, one of the following conditions holds:

$1^{\epsilon}: R_{\epsilon \infty}=R_{\epsilon k}$ for some positive integer $k$ and $D / R_{\epsilon \infty}$ is a cycle.

$2^{\epsilon}: R_{\epsilon \infty}=R_{\epsilon k}$ for some positive integer $k$ and $D / R_{\epsilon \infty}$ is a chain.

$3^{\epsilon}: R_{\epsilon \infty}=R_{\epsilon k}$ for some positive integer $k$ and $D / R_{\epsilon \infty}$ is a regular directed tree with in-degree $>1$ and out-degree 1 in the case $\epsilon=-$ and with in-degree 1 and out-degree $>1$ in the case $\epsilon=+$.

$4^{\epsilon}: R_{\epsilon \infty} \neq R_{\epsilon k}$ for any positive integer $k$ and $D / R_{\epsilon \infty}$ is a chain.

$5^{\epsilon}: R_{\epsilon \infty} \neq R_{\epsilon k}$ for every positive integer $k$ and $D / R_{\epsilon \infty}$ is a regular directed tree with in-degree $>1$ and out-degree 1 in the case $\epsilon=-$ and with in-degree 1 and out-degree $>1$ in the case $\epsilon=+$.

It is quite natural to ask which pairs $\left(i^{-}, j^{+}\right), 1 \leq i, j \leq 5$, of these properties can occur simultaneously for locally finite connected transitive digraphs $D$ ? In this paper we proof the following Table Theorem which answers this question.

Theorem 1.1. Let $D$ be a locally finite connected transitive digraph. In the following table the symbol $Y$ at the entry $i^{-}, j^{+}$indicates that $D$ can have properties $i^{-}$and $j^{+}$ simultaneously; $N$ means that $D$ cannot have both properties simultaneously.

\begin{tabular}{|c|c|c|c|c|c|}
\hline & $1^{+}$ & $2^{+}$ & $3^{+}$ & $4^{+}$ & $5^{+}$ \\
\hline $1^{-}$ & $Y$ & $N$ & $N$ & $N$ & $N$ \\
\hline $2^{-}$ & $N$ & $Y$ & $N$ & $N$ & $N$ \\
\hline $3^{-}$ & $N$ & $N$ & $N$ & $Y$ & $N$ \\
\hline $4^{-}$ & $N$ & $N$ & $Y$ & $N$ & $N$ \\
\hline $5^{-}$ & $N$ & $N$ & $N$ & $N$ & $Y$ \\
\hline
\end{tabular}

We also investigate the same question for digraphs $D$ from natural subclasses of the class of locally finite connected transitive digraphs. In Section 4 we consider the following subclasses: Cayley digraphs of finitely generated groups, the class of locally finite connected transitive digraphs with infinitely many ends, locally finite connected transitive digraphs containing certain directed subtrees, and locally finite connected highly arc transitive digraphs. 


\section{General properties of reachability relations}

In this section we present some general facts on reachability relations on digraphs, which are used (often implicitly) in the sequel. To do that we need some additional definitions:

Let $D$ be a digraph. A sequence $\left(v_{0}, \ldots, v_{n}\right), n \geq 1$, of pairwise different vertices of $D$ satisfying $\left(v_{i}, v_{i+1}\right) \in E(D)$ for all $0 \leq i<n$ is a directed path in $D$ of length $n$. For any positive integer $i$, the digraph $D^{i}$ is defined by $V\left(D^{i}\right)=V(D)$ and $E\left(D^{i}\right)=\{(u, v) \mid u \neq$ $v$ and there exists a directed path

of length $\leq i$ in $D$ with initial vertex $u$ and terminal vertex $v\}$. For any positive integer $i$, the graph $\bar{D}^{i}$ is obtained from $\bar{D}$ by inserting edges between any two different vertices which are at distance at most $i$ in $\bar{D}$.

A walk of a digraph is closed if its initial vertex and its terminal vertex coincide. For a walk $W=\left(v_{0}, e_{1}^{\epsilon_{1}}, v_{1}, \ldots, v_{n-1}, e_{n}^{\epsilon_{n}}, v_{n}\right)$ of a digraph $D$, the walk $W^{-1}$ of $D$ is defined by $W^{-1}:=\left(v_{n}, e_{n}^{-\epsilon}, v_{n-1}, \ldots, v_{1}, e_{1}^{-\epsilon}, v_{0}\right)$. If $l(W)=0$ we set $W^{-1}=W$. If $W=\left(v_{0}, e_{1}^{\epsilon_{1}}, v_{1}, \ldots, v_{n-1}, e_{n}^{\epsilon_{n}}, v_{n}\right)$ and $W^{\prime}=\left(u_{0}, f_{1}^{\sigma_{1}}, u_{1}, \ldots, u_{m-1}, f_{m}^{\sigma_{m}}, u_{m}\right)$ are walks of a digraph $D$ such that $v_{n}=u_{0}$, then the walk $W W^{\prime}$ is called the concatenation of $W$ and $W^{\prime}$ and is defined by

$$
W W^{\prime}:=\left(v_{0}, e_{1}^{\epsilon_{1}}, v_{1}, \ldots, v_{n-1}, e_{n}^{\epsilon_{n}}, v_{n}=u_{0}, f_{1}^{\sigma_{1}}, u_{1}, \ldots, u_{m-1}, f_{m}^{\sigma_{m}}, u_{m}\right)
$$

(we set $W:=W W^{\prime}$ if $l\left(W^{\prime}\right)=0$ and $W^{\prime}:=W W^{\prime}$ if $l(W)=0$ ).

For a digraph $D$, the digraph $D^{*}$ is defined by $V\left(D^{*}\right)=V(D)$ and $E\left(D^{*}\right)=\{(u, v) \mid$ $(v, u) \in E(D)\}$. Note that, for any $a \in \mathbf{Z}_{\leq 0} \cup\{-\infty\}$ and any $b \in \mathbf{Z}_{\geq 0} \cup\{+\infty\}$, the reachability relation $R_{a, b}^{D}$ coincides with the reachability relation $R_{-b,-a}^{D^{*}}$ (on $V(D)=$ $\left.V\left(D^{*}\right)\right)$.

As usual, a digraph $D_{1}$ is contained in a digraph $D_{2}$ if $V\left(D_{1}\right) \subseteq V\left(D_{2}\right)$ and $E\left(D_{1}\right) \subseteq$ $E\left(D_{2}\right)$.

In the sequel we present some basic properties of reachability relations in digraphs.

Proposition 2.1 follows immediately from the above definitions.

Proposition 2.1. Let $D$ be a digraph, let $a \in \mathbf{Z}_{\leq 0} \cup\{-\infty\}$ and $b \in \mathbf{Z}_{\geq 0} \cup\{+\infty\}$. If in addition $a^{\prime} \in \mathbf{Z}_{\leq 0} \cup\{-\infty\}$ and $b^{\prime} \in \mathbf{Z}_{\geq 0} \cup\{+\infty\}$, then the following assertions hold.

(1) The equivalence relation on $\bar{V}(D)$ generated by $R_{a, b}$ and $R_{a^{\prime}, b^{\prime}}$ coincides with $R_{\min \left\{a, a^{\prime}\right\}, \max \left\{b, b^{\prime}\right\}}$,

(2) The union of all equivalence classes with respect to $R_{a, b}^{D}$ contained in an arbi-

trary fixed equivalence class with respect to $R_{a^{\prime}, b^{\prime}}^{D / R_{a, b}^{D}}$ is an equivalence class with respect to $R_{a+a^{\prime}, b+b^{\prime}}^{D}$

Proposition 2.2. Let $D$ be a connected transitive digraph, let $a \in \mathbf{Z}_{\leq 0} \cup\{-\infty\}, b \in$ $\mathbf{Z}_{\geq 0} \cup\{+\infty\}$ and suppose $\left|V\left(D / R_{a, b}\right)\right|>1$. Then $u \notin R_{a, b}(v)$ for any vertex $u$ with $(u, v) \in E(D)$.

Proof. Assume there exists $(u, v) \in E(D)$ with $u R_{a, b} v$. Since $u \neq v$ it follows that $a<0$ or $b>0$. Since $D$ is a connected transitive digraph and $\left|V\left(D / R_{a, b}\right)\right|>1$, there exist 
$\left(u, u^{\prime}\right) \in E(D)$ and $\left(v^{\prime}, v\right) \in E(D)$ such that $u^{\prime} \notin R_{a, b}(u)=R_{a, b}(v)$ and $v^{\prime} \notin R_{a, b}(v)=$ $R_{a, b}(u)$. If $a<0$ we obtain that $\left(v,(u, v)^{-1}, u,\left(u, u^{\prime}\right), u^{\prime}\right) \in \mathcal{R}_{a, b}$; if $b>0$ we obtain that $\left(u,(u, v), v,\left(v^{\prime}, v\right)^{-1}, v^{\prime}\right) \in \mathcal{R}_{a, b}$. Thus $u^{\prime} \in R_{a, b}(v)$ or $v^{\prime} \in R_{a, b}(u)$, a contradiction.

Let $D$ be a digraph and let $a \in \mathbf{Z}_{\leq 0} \cup\{-\infty\}, b \in \mathbf{Z}_{\geq 0} \cup\{+\infty\}$. For $u, v \in$ $V(D)$ set $u S_{a} v$ if either $a=0$ and $u=v$ or $a \neq 0$ and there exists a walk $(u=$ $\left.v_{0}, e_{1}^{\epsilon_{1}}, v_{1}, \ldots, v_{2 n-1}, e_{2 n}^{\epsilon_{2 n}}, v_{2 n}=v\right)$ in $D$ with $n \leq-a$ such that $\epsilon_{1}, \ldots, \epsilon_{n}=-1$ and $\epsilon_{n+1}, \ldots, \epsilon_{2 n}=1$. Analogously, for $u, v \in V(D)$ put $u S_{b} v$ if either $b=0$ and $u=v$ or $b \neq 0$ and there exists a walk $\left(u=v_{0}, e_{1}^{\epsilon_{1}}, v_{1}, \ldots, v_{2 n-1}, e_{2 n}^{\epsilon_{2 n}}, v_{2 n}=v\right)$ of $D$ with $n \leq b$ such that $\epsilon_{1}, \ldots, \epsilon_{n}=1$ and $\epsilon_{n+1}, \ldots, \epsilon_{2 n}=-1$.

Proposition 2.3. Let $D$ be a digraph, let $a \in \mathbf{Z}_{\leq 0} \cup\{-\infty\}$ and let $b \in \mathbf{Z}_{\geq 0} \cup\{+\infty\}$. Then the following assertions hold:

(1) If the out-degree of every vertex of $D$ is $\geq 1$, then $R_{a, 0}$ is the minimal equivalence relation on $V(D)$ containing the relation $S_{a}$.

(2) If the in-degree of every vertex of $D$ is $\geq 1$, then $R_{0, b}$ is the minimal equivalence relation on $V(D)$ containing the relation $S_{b}$.

(3) If both the out-degree and the in-degree of every vertex of $D$ are $\geq 1, R_{a, b}$ is the minimal equivalence relation on $V(D)$ containing the relation $S_{a}$ as well as the relation $S_{b}$.

Proof. To prove (1) note first that $S_{a}$ is contained in $R_{a, 0}$. On the other hand, assume that $u R_{a, 0} v$ and $u \neq v$. Then, by definition, there exists a walk $W=(u=$ $\left.v_{0}, e_{1}^{\epsilon_{1}}, v_{1}, \ldots, v_{n-1}, e_{n}^{\epsilon_{n}}, v_{n}=v\right)$ in $D$ such that, for any $0 \leq j \leq n$, the height of the $(0, j)$-subwalk $W_{0, j}$ of $W$ is non-positive and not smaller than $a$. Since the out-degree of every vertex of $D$ is $\geq 1$, for each $j, 0 \leq j \leq n$, either $\operatorname{ht}\left(W_{0, j}\right)=0$ (in this case we define $\left.w_{j}:=v_{j}\right)$ or there exists a walk $\left(v_{j}=v_{j, 0}, e_{j, 1}, v_{j, 1}, \ldots, v_{j,-\mathrm{ht}\left(W_{0, j}\right)-1}, e_{j,-\mathrm{ht}\left(W_{0, j}\right)}, v_{j,-\mathrm{ht}\left(W_{0, j}\right)}\right)$, where $e_{j, 1}, \ldots, e_{j,-\mathrm{ht}\left(W_{0, j}\right)} \in E(D)$ (in this case we set $\left.w_{j}:=v_{j,-\mathrm{ht}\left(W_{0, j}\right)}\right)$. Now $w_{i} S_{a} w_{i+1}$ for every $i, 0 \leq i \leq n-1$, since $-\operatorname{ht}\left(W_{0, j}\right)<-a$. Since $w_{0}=v_{0}=u$ and $w_{n}=v_{n}=v$, it follows that $u$ and $v$ are equivalent with respect to the minimal equivalence relation on $V(D)$ containing $S_{a}$. Thus assertion (1) holds. Assertion (2) can be shown analogously. Since $R_{a, b}$ is the minimal equivalence relation on $V(D)$ containing $R_{a, 0}$ and $R_{0, b}$, assertion (3) immediately follows from (1) and (2).

Corollary 2.4. Let $D$ be a digraph and let $a \in \mathbf{Z}_{\leq 0} \cup\{-\infty\}, b \in \mathbf{Z}_{\geq 0} \cup\{+\infty\}$. Then the following assertions hold.

(1) If the in-degree of every vertex of $D$ is $\geq 1$ and $R_{a, k}=R_{a,+\infty}$ for some non-negative integer $k$, then $R_{0, k-a}=R_{0,+\infty}$.

(2) If the out-degree of every vertex of $D$ is $\geq 1$ and $R_{-k, b}=R_{-\infty, b}$ for some nonnegative integer $k$, then $R_{-k-b, 0}=R_{-\infty, 0}$. 
Proof. We prove assertion (2). Then assertion (1) obviously holds, since it is equivalent to assertion (2) applied to the digraph $D^{*}$.

Of course, $R_{-k-b, 0} \subseteq R_{-\infty, 0}$. By Proposition 2.3 it is sufficient to show that $S_{-\infty} \subseteq$ $R_{-k-b, 0}$ to prove that $R_{-\infty, 0} \subseteq R_{-k-b, 0}$. Assume $u, v \in V(D)$ and $u S_{-\infty} v$. Of course, $u R_{-k-b, 0} v$ if $u=v$. Suppose $u \neq v$. Then, by the definition of $S_{-\infty}$, for some positive integer $n$ there exist walks $U=\left(u=u_{0}, e_{1}^{-1}, u_{1}, \ldots, e_{n}^{-1}, u_{n}\right)$ and $V=\left(v=v_{0}, e_{1}^{-1}, v_{1}, \ldots, e_{n}^{\prime-1}\right.$, $v_{n}$ ) of $D$ such that $u_{n}=v_{n}$. If $n \leq k+b$ it follows that $u R_{-k-b, 0} v$. Suppose $n>k+b$. Then $U_{b, n} V_{b, n}^{-1} \in \mathcal{R}_{-\infty, 0}$. Since $R_{-\infty, b}=R_{-k, b}$ it follows that there exists a walk $W \in \mathcal{R}_{-k, b}$ with initial vertex $u_{b}$ and terminal vertex $v_{b}$. Now $U_{0, b} W V_{0, b}^{-1} \in \mathcal{R}_{-k-b, 0}$ and therefore $u R_{-k-b, 0} v$, which completes the proof.

Proposition 2.5. Let $D$ be a digraph and let $a \in \mathbf{Z}_{\leq 0} \cup\{-\infty\}, b \in \mathbf{Z}_{\geq 0} \cup\{+\infty\}$. Then the following assertions hold:

(1) Conditions $(1 a)-(1 c)$ are equivalent:

(1a) $R_{a-1, b}=R_{a, b}$

(1b) $R_{a, b}=R_{-\infty, b}$,

(1c) the out-degree of every vertex of $D / R_{a, b}$ is $\leq 1$.

(2) Conditions (2a) $-(2 c)$ are equivalent:

(2a) $R_{a, b+1}=R_{a, b}$,

(2b) $R_{a, b}=R_{a,+\infty}$,

(2c) the in-degree of every vertex of $D / R_{a, b}$ is $\leq 1$.

(3) Conditions (3a) - (3c) are equivalent:

(3a) $R_{a-1, b+1}=R_{a, b}$,

(3b) $R_{a, b}=R_{-\infty,+\infty}$

(3c) both, the in-degree and the out-degree of every vertex of $D / R_{a, b}$ are $\leq 1$.

Proof. We only prove assertion (1), since assertions (2) and (3) can be proved analogously. Obviously, (1c) implies (1b) while (1b) implies (1a). Suppose that (1a) holds. Then, setting $(a, b)=(-1,0)$ and $\left(a^{\prime}, b^{\prime}\right)=(0,0)$ in Proposition 2.1(1), we obtain that $R_{-1,0}^{D / R_{a, b}^{D}}=R_{0,0}^{D / R_{a, b}^{D}}$. This implies that the out-degree of every vertex of the digraph $D / R_{a, b}$ is $\leq 1$. Thus (1a) implies (1c) and (1) holds.

Corollary 2.6. Let $D$ be a digraph and let $a \in \mathbf{Z}_{\leq 0} \cup\{-\infty\}, b \in \mathbf{Z}_{\geq 0} \cup\{+\infty\}$. Then the following assertions hold:

(1) The out-degree of every vertex of $D / R_{-\infty, b}$ is $\leq 1$.

(2) The in-degree of every vertex of $D / R_{a,+\infty}$ is $\leq 1$.

(3) Both the in-degree and the out-degree of every vertex of $D / R_{-\infty,+\infty}$ are $\leq 1$.

Corollary 2.7. Let $D$ be a digraph and let $a \in \mathbf{Z}_{\leq 0} \cup\{-\infty\}, b \in \mathbf{Z}_{>0} \cup\{+\infty\}$. Then the following assertions hold: 
(1) $D / R_{-\infty, b}$ is one of the following graphs: a cycle, a chain or a regular directed tree with in-degree $>1$ and out-degree 1.

(2) $D / R_{a,+\infty}$ is one of the following graphs: a cycle, a chain or a regular directed tree with in-degree 1 and out-degree $>1$.

(3) $D / R_{-\infty,+\infty}$ is either a cycle or a chain.

The next result gives a simple condition under which $D / R_{-\infty,+\infty}$ is a cycle.

Corollary 2.8. Let $D$ be a connected transitive digraph. Suppose there exists a closed walk $W$ in $D$ with $\mathrm{ht}(W) \neq 0$. Then $D / R_{-\infty,+\infty}$ is a cycle and $\left|V\left(D / R_{-\infty,+\infty}\right)\right|$ divides $\operatorname{ht}(W)$.

Proposition 2.9. Let $D$ be a transitive digraph. Suppose there exists a closed walk $W$ of $D$ with $\mathrm{ht}(W) \neq 0$. Put $a_{W}:=\min \left\{\mathrm{ht}\left(W_{0, j}\right): 0 \leq j \leq l(W)\right\}$ and $b_{W}:=\max \left\{\operatorname{ht}\left(W_{0, j}\right)\right.$ : $0 \leq j \leq l(W)\}$. Then $R_{a, b}=R_{-\infty,+\infty}$ for any non-positive integer a and any non-negative integer $b$ with $b-a \geq b_{W}-a_{W}$.

Proof. Since $D$ is transitive, there exists a closed walk $W_{x}$ of length $l(W)$ for every $x \in$ $V(D)$ whose initial vertex and terminal vertex coincides with $x$, such that ht $\left(\left(W_{x}\right)_{0, j}\right)=$ $\operatorname{ht}\left(W_{0, j}\right)$ for all $j, 0 \leq j \leq l(W)$.

Let $a$ be an arbitrary non-positive integer and let $b$ be an arbitrary non-negative integer with $b-a \geq b_{W}-a_{W}$. For a walk $U$ in $D$ with $h t(U)=0$, set

$$
\mathcal{H}(U):=\left\{j: 0 \leq j \leq l(U) \text { and either } \operatorname{ht}\left(U_{0, j}\right)<a \text { or } \operatorname{ht}\left(U_{0, j}\right)>b\right\} .
$$

Proceeding by induction on $|\mathcal{H}(U)|$ we prove that the initial vertex and the terminal vertex of an arbitrary walk $U$ of $D$ with ht $(U)=0$ are $R_{a, b}$-equivalent.

If $|\mathcal{H}(U)|=0$, then $U \in \mathcal{R}_{a, b}$, and the initial vertex and the terminal vertex of $U$ are $R_{a, b}$-equivalent by definition.

Assume that there exists an integer $i, 0 \leq i \leq l(U)$, with $h t\left(U_{0, i}\right)<a$. Then we can write $U=U^{\prime} U^{\prime \prime} U^{\prime \prime \prime}$ where $l\left(U^{\prime}\right)<i<l\left(U^{\prime} U^{\prime \prime}\right), \operatorname{ht}\left(U^{\prime}\right)=a=\operatorname{ht}\left(U^{\prime} U^{\prime \prime}\right)$ and $\operatorname{ht}\left(U_{0, j}\right)<a$ for all $j, l\left(U^{\prime}\right)<j<l\left(U^{\prime} U^{\prime \prime}\right)$. Let $x^{\prime}$ be the initial vertex of the walk $U^{\prime \prime}$, and $x^{\prime \prime}$ be the terminal vertex of the walk $U^{\prime \prime}$. Since $D$ is a transitive digraph and $E(D) \neq \emptyset$, there exists a walk $W^{\prime}$ in $D$ with initial vertex $x^{\prime}$ such that ht $\left(W^{\prime}\right)=l\left(W^{\prime}\right)=-a_{W}$ if $\operatorname{ht}(W)>0$ and $\operatorname{ht}\left(W^{\prime}\right)=l\left(W^{\prime}\right)=b_{W}$ if $\operatorname{ht}(W)<0$. Analogously, there exists a walk $W^{\prime \prime}$ in $D$ with initial vertex $x^{\prime \prime}$ such that ht $\left(W^{\prime \prime}\right)=l\left(W^{\prime \prime}\right)=b_{W}-\operatorname{ht}(W)$ if $h t(W)>0$ and ht $\left(W^{\prime \prime}\right)=l\left(W^{\prime \prime}\right)=$ $-a_{W}+\operatorname{ht}(W)$ if $\operatorname{ht}(W)<0$. Let $y^{\prime}$ be the terminal vertex of the walk $W^{\prime}$, and let $y^{\prime \prime}$ be the terminal vertex of the walk $W^{\prime \prime}$. Define $\tilde{U}:=U^{\prime} W^{\prime} W_{y^{\prime}}\left(W^{\prime}\right)^{-1} U^{\prime \prime} W^{\prime \prime}\left(W_{y^{\prime \prime}}\right)^{-1}\left(W^{\prime \prime}\right)^{-1} U^{\prime \prime \prime}$ if $\operatorname{ht}(W)>0$, and set $\tilde{U}:=U^{\prime} W^{\prime}\left(W_{y^{\prime}}\right)^{-1}\left(W^{\prime}\right)^{-1} U^{\prime \prime} W^{\prime \prime} W_{y^{\prime \prime}}\left(W^{\prime \prime}\right)^{-1} U^{\prime \prime \prime}$ if $h t(W)<0$. Then $\operatorname{ht}(\tilde{U})=0$ and $|\mathcal{H}(\tilde{U})|<|\mathcal{H}(U)|$. Thus, by our induction hypothesis, the initial vertex of $U$ (which coincides with the initial vertex of $\tilde{U}$ ) and the terminal vertex of $U$ (which coincides with the terminal vertex of $\tilde{U}$ ) are $R_{a, b}$-equivalent.

Assume now that there exists an integer $i, 0 \leq i \leq l(U)$ with $h t\left(U_{0, i}\right)>b$. Then we can write $U=U^{\prime} U^{\prime \prime} U^{\prime \prime \prime}$ where $l\left(U^{\prime}\right)<i<l\left(U^{\prime} U^{\prime \prime}\right)$, ht $\left(U^{\prime}\right)=b=h t\left(U^{\prime} U^{\prime \prime}\right)$ and 
ht $\left(U_{0, j}\right)>b$ for all $j, l\left(U^{\prime}\right)<j<l\left(U^{\prime} U^{\prime \prime}\right)$. Let $x^{\prime}$ be the initial vertex of the walk $U^{\prime \prime}$, and $x^{\prime \prime}$ be the terminal vertex of the walk $U^{\prime \prime}$. Since $D$ is a transitive digraph and $E(D) \neq \emptyset$, there exists a walk $W^{\prime}$ of $D$ with initial vertex $x^{\prime}$ such that $h t\left(W^{\prime}\right)=$ $-l\left(W^{\prime}\right)=a_{W}$ in the case $\mathrm{ht}(W)>0$ and $\mathrm{ht}\left(W^{\prime}\right)=-l\left(W^{\prime}\right)=-b_{W}$ in the case $\mathrm{ht}(W)<0$. Analogously, there exists a walk $W^{\prime \prime}$ in $D$ with initial vertex $x^{\prime \prime}$ such that $\operatorname{ht}\left(W^{\prime \prime}\right)=$ $-l\left(W^{\prime \prime}\right)=-b_{W}+\operatorname{ht}(W)$ if $\operatorname{ht}(W)>0$ and $\operatorname{ht}\left(W^{\prime \prime}\right)=-l\left(W^{\prime \prime}\right)=a_{W}-\operatorname{ht}(W)$ if $\operatorname{ht}(W)<$ 0 . Let $y^{\prime}$ be the terminal vertex of the walk $W^{\prime}$, and let $y^{\prime \prime}$ be the terminal vertex of the walk $W^{\prime \prime}$. Define $\tilde{U}:=U^{\prime} W^{\prime}\left(W_{y^{\prime}}\right)^{-1}\left(W^{\prime}\right)^{-1} U^{\prime \prime} W^{\prime \prime} W_{y^{\prime \prime}}\left(W^{\prime \prime}\right)^{-1} U^{\prime \prime \prime}$ if $\operatorname{ht}(W)>0$ and set $\tilde{U}:=U^{\prime} W^{\prime} W_{y^{\prime}}\left(W^{\prime}\right)^{-1} U^{\prime \prime} W^{\prime \prime}\left(W_{y^{\prime \prime}}\right)^{-1}\left(W^{\prime \prime}\right)^{-1} U^{\prime \prime \prime}$ if $\operatorname{ht}(W)<0$. Then $\operatorname{ht}(\tilde{U})=0$ and $|\mathcal{H}(\tilde{U})|<|\mathcal{H}(U)|$. Thus, by our induction hypothesis, the initial vertex of $U$ (which coincides with the initial vertex of $\tilde{U}$ ) and the terminal vertex of $U$ (which coincides with the terminal vertex of $\tilde{U}$ ) are $R_{a, b}$-equivalent.

We say that a connected digraph $D$ has property $\mathbf{Z}$ if there exists a homomorphism of $D$ onto a chain, i.e. a mapping $\chi$ of $V(D)$ onto the set $\mathbf{Z}$ of integers such that $\chi(v)=\chi(u)+1$ for any $(u, v) \in E(D)$. Note that, if the digraph $D$ with property $\mathbf{Z}$ admits a vertextransitive group of automorphisms $G$, then $\chi$ induces a natural homomorphism from $G$ onto $\mathbf{Z}$.

The next result is more or less obvious and we present it without proof (see also [8]). Observation. Let $D$ be a connected digraph with $|V(D)|>1$. Then $D$ has property $\mathbf{Z}$ if and only if $\mathrm{ht}(W)=0$ for any closed walk $W$ of $D$.

As a consequence of this observation and Proposition 2.9 we have the following result.

Proposition 2.10. Let $D$ be a connected transitive digraph without property $\mathbf{Z}$. Then there exists a non-negative integer $c$ such that $R_{a, b}=R_{-\infty,+\infty}$ for any non-positive integer $a$ and any non-negative integer $b$ with $b-a \geq c$.

The following result immediately follows from Proposition 2.2.

Proposition 2.11. Let $D$ be a connected transitive digraph. Then the following conditions are equivalent.

(1) For some $a \in \mathbf{Z}_{\leq 0} \cup\{-\infty\}$ and some $b \in \mathbf{Z}_{\geq 0} \cup\{+\infty\}$ the digraph $D / R_{a, b}$ has property $\mathbf{Z}$.

$\mathrm{Z}$.

(2) For any $a \in \mathbf{Z}_{\leq 0} \cup\{-\infty\}$ and any $b \in \mathbf{Z}_{\geq 0} \cup\{+\infty\}$ the digraph $D / R_{a, b}$ has property

Corollary 2.12. Let $D$ be a connected transitive digraph and let $a \in \mathbf{Z}_{\leq 0} \cup\{-\infty\}, b \in$ $\mathbf{Z}_{\geq 0} \cup\{+\infty\}$. Then the following assertions are equivalent:

(1) $D / R_{-\infty, b}$ is infinite (by Corollary 2.7 this means that $D / R_{-\infty, b}$ is either a chain or a regular directed tree with in-degree $>1$ and out-degree 1$)$.

(2) $D / R_{a,+\infty}$ is infinite (by Corollary 2.7 this means that $D / R_{a,+\infty}$ is either a chain or a regular directed tree with in-degree 1 and out-degree $>1)$.

(3) $D / R_{-\infty,+\infty}$ is infinite (by Corollary 2.7 it means that $D / R_{-\infty,+\infty}$ is a chain).

(4) D has property $\mathbf{Z}$. 
Proof. If one of (1), (2), (3) or (4) holds, then the digraphs $D / R_{-\infty, b}, D / R_{a,+\infty}$, $D / R_{-\infty,+\infty}$ or $D / R_{0,0}=D$ have property $\mathbf{Z}$, respectively. Thus the result follows from Proposition 2.11.

Proposition 2.13. Let $D$ be a connected transitive digraph and let $a \in \mathbf{Z}_{\leq 0} \cup\{-\infty\}$, $b \in \mathbf{Z}_{\geq 0} \cup\{+\infty\}$. Then the following assertions hold:

(1) If $R_{a, k+1} \neq R_{a, k}$ for every positive integer $k$, then $D$ contains a regular tree with in-degree 2 and out-degree 1 .

(2) If $R_{-k-1, b} \neq R_{-k, b}$ for every positive integer $k$, then $D$ contains a regular tree with in-degree 1 and out-degree 2 .

Proof. We only prove assertion (2) since (1) is equivalent to (2), formulated for the digraph $D^{*}$.

Assume $R_{-k-1, b} \neq R_{-k, b}$ for every positive integer $k$. Then assertion (1) of Proposition 2.1 implies that $R_{-k-1} \neq R_{-k}$ for every positive integer $k$. Furthermore, by Proposition 2.10 and Corollary 2.12 the digraph $D$ has property $\mathbf{Z}$ and the digraph $D / R_{-\infty,+\infty}$ is a chain. In particular, the vertex-transitive group of automorphisms $\operatorname{Aut}(D)$ induces an infinite cyclic group of automorphisms of $D / R_{-\infty,+\infty}$.

It is easy to see that any transitive digraph with infinite out-degree contains a regular tree with in-degree 1 and out-degree 2 . Thus without loss of generality we can assume that $\operatorname{deg}^{+}(D)$ is finite. Now arguments used in the proof of Theorem 4.2 in [12] or in the proof of Theorem 4.12 in [8] can be easily adapted to prove that $D$ contains a regular tree with in-degree 1 and out-degree 2.

For the convenience of the reader we roughly outline the arguments of the proof in [8] here. The digraph $D$ in consideration has property $\mathbf{Z}$. Since $D$ is transitive with finite $\operatorname{deg}^{+}(D)$ and $R_{-k-1, b} \neq R_{-k, b}$ for every positive integer $k$, we can find, for any vertex $u$ of $D$, edges $\left(u, u^{\nearrow}\right)$ and $\left(u, u^{\searrow}\right)$ of $D$ such that there exist arbitrarily long directed paths $\left(v_{0}, v_{1}, \ldots, v_{l}\right)$ and $\left(w_{0}, w_{1}, \ldots, w_{l}\right)$ in $D$ with $v_{0}=w_{0}=u, v_{1}=u^{\nearrow}, w_{1}=$ $u^{\searrow}$ and $v_{l} \notin R_{-l+1,0}\left(w_{l}\right)$. By the choice of $\left(u, u^{\nearrow}\right)$ and $\left(u, u^{\searrow}\right)$, for any directed paths $\left(v_{0}^{\prime}, v_{1}^{\prime}, \ldots, v_{m}^{\prime}\right)$ and $\left(w_{0}^{\prime}, w_{1}^{\prime}, \ldots, w_{n}^{\prime}\right)$ in $D$ with $v_{0}^{\prime}=w_{0}^{\prime}=u, v_{1}^{\prime}=u^{\prime}$ and $w_{1}^{\prime}=u^{\searrow}$, we have $\left\{v_{1}^{\prime}, \ldots, v_{m}^{\prime}\right\} \cap\left\{w_{1}^{\prime}, \ldots, w_{n}^{\prime}\right\}=\emptyset$. Fix an arbitrary vertex $u$ of $D$, and define a subgraph $T_{u}$ of $D$ by $V\left(T_{u}\right)=\{u\} \cup\left\{\left(\ldots\left(u^{\epsilon_{1}}\right) \ldots\right)^{\epsilon_{t}}: t \geq 1\right.$ and $\epsilon_{s} \in\{\nearrow, \searrow\}$ for each $\left.s, 1 \leq s \leq t\right\}$ and $E\left(T_{u}\right)=\left\{\left(u, u^{\nearrow}\right),\left(u, u^{\searrow}\right)\right\} \cup\left\{\left(\left(\ldots\left(u^{\epsilon_{1}}\right) \ldots\right)^{\epsilon_{t}},\left(\left(\ldots\left(u^{\epsilon_{1}}\right) \ldots\right)^{\epsilon_{t}}\right)^{\epsilon_{t+1}}\right): t \geq 1\right.$ and $\epsilon_{s} \in\{\nearrow$ $, \searrow\}$ for each $s, 1 \leq s \leq t+1\}$. The subgraph $T_{u}$ is a directed subtree of $D$ such that the in-degree of every vertex of $T_{u}$ different from $u$ is 1 (the in-degree of $u$ in $T_{u}$ is 0 ) and the out-degree of every vertex of $T_{u}$ is 2 . Since $D$ is a transitive digraph, the result follows. 


\section{Proof of Theorem 1.1}

Obviously, if $D$ is a digraph satisfying the pair of conditions $\left(i^{-}, j^{+}\right)$, where $1 \leq i, j \leq 5$, then the digraph $D^{*}$ satisfies the pair of conditions $\left(j^{-}, i^{+}\right)$. Thus, for any $1 \leq i, j \leq 5$, the pair of possibilities $\left(i^{-}, j^{+}\right)$is realized by some connected transitive digraph if and only if the same holds for the pair $\left(j^{-}, i^{+}\right)$. Hence it is sufficient to investigate which pairs $\left(i^{-}, j^{+}\right)$with $1 \leq i \leq j \leq 5$ are realized for connected transitive digraphs.

$\left(\mathbf{1}^{-}, \mathbf{1}^{+}\right)$: Obviously, any cycle (and, more generally, any finite connected transitive digraph) has properties $1^{-}$and $1^{+}$simultaneously. Moreover, by Proposition 2.9 and Corollaries 2.8, 2.12 and 2.7, any connected transitive digraph containing a closed walk of non-zero height has properties $1^{-}$and $1^{+}$simultaneously.

$\left(\mathbf{1}^{-}, \mathbf{2}^{+}\right),\left(\mathbf{1}^{-}, \mathbf{3}^{+}\right),\left(\mathbf{1}^{-}, \mathbf{4}^{+}\right),\left(\mathbf{1}^{-}, \mathbf{5}^{+}\right)$: Properties $2^{+}, 3^{+}, 4^{+}$and $5^{+}$all imply that $D / R_{0,+\infty}$ is an infinite graph. Then it follows from Corollary 2.12 that also $D / R_{-\infty, 0}$ is infinite, a contradiction to property $1^{-}$. Therefore all these pairs of properties cannot occur simultaneously.

$\left(\mathbf{2}^{-}, \mathbf{2}^{+}\right)$: If $D$ is a chain, then of course $D$ has both properties.

$\left(\mathbf{2}^{-}, \mathbf{3}^{+}\right),\left(\mathbf{2}^{-}, \mathbf{4}^{+}\right),\left(\mathbf{2}^{-}, \mathbf{5}^{+}\right)$: Assume that a connected transitive digraph $D$ has property $2^{-}$and simultaneously one of the properties $3^{+}, 4^{+}$or $5^{+}$. By $2^{-}, R_{-\infty, 0}=R_{-k, 0}$ for some positive integer $k$. We show first that $R_{-\infty, 0} \subseteq R_{0, k}$. Let $u R_{-\infty, 0} v$. Since $D$ is transitive and $2^{-}$is satisfied, there are walks $U=\left(u=u_{0}, e_{1}, u_{1}, \ldots, e_{k}, u_{k}\right)$ and $V=\left(v=v_{0}, e_{1}^{\prime}, v_{1}, \ldots, e_{k}^{\prime}, v_{k}\right)$ in $D$ such that $u_{k} R_{-\infty, 0} v_{k}$. In addition $2^{-}$implies that $u_{k} R_{-k, 0} v_{k}$, i.e. there exists a walk $W \in \mathcal{R}_{-k, 0}$ with initial vertex $u_{k}$ and terminal vertex $v_{k}$. Now $U W V^{-1} \in \mathcal{R}_{0, k}$ and, consequently, $u R_{0, k} v$. Thus $R_{-\infty, 0} \subseteq R_{0, k}$. On the other hand, $D / R_{-\infty, 0}$ is a chain by property $2^{-}$. Hence, by assertion (2) of Proposition 2.1, $R_{-\infty, 0}=R_{-\infty,+\infty}$. Therefore, by assertion (1) of Proposition 2.1, $R_{-\infty, 0}=$ $R_{0, k} \subseteq R_{0,+\infty} \subseteq R_{-\infty,+\infty}$. In particular, $R_{0, k}=R_{0,+\infty}$ and, consequently, neither $4^{+}$nor $5^{+}$can be satisfied. Furthermore, since $R_{-\infty, 0}=R_{0,+\infty}$, it follows from $2^{-}$that $D / R_{0,+\infty}$ is a chain. Thus $3^{+}$also can not be satisfied. We conclude that, for connected transitive digraphs, $2^{-}$can not occur simultaneously with any of the properties $3^{+}, 4^{+}, 5^{+}$.

$\left(3^{-}, 3^{+}\right),\left(3^{-}, 5^{+}\right)$: Assume that, for a connected transitive digraph $D, 3^{-}$and one of properties $3^{+}$or $5^{+}$hold simultaneously. By $3^{-}, R_{-\infty, 0}=R_{-k, 0}$ for some positive integer $k$. Analogously to the case $\left(2^{-}, 3^{+}\right)$it is possible to show that $R_{-\infty, 0} \subseteq R_{0, k} \subseteq R_{0,+\infty}$. By assertion (1) of Proposition 2.1, it follows that $R_{-\infty,+\infty}=R_{0,+\infty}$. Since one of the properties $3^{+}$or $5^{+}$holds, we also obtain that $D / R_{-\infty,+\infty}=D / R_{0,+\infty}$ is a regular directed tree with in-degree 1 and out-degree $>1$. But this contradicts assertion (3) of Corollary 2.7. Therefore, for connected transitive digraphs, property $3^{-}$excludes properties $3^{+}$and $5^{+}$. $\left(3^{-}, 4^{+}\right)$: Any regular directed tree with out-degree 1 and in-degree $>1$ has properties $3^{-}$and $4^{+}$.

$\left(4^{-}, 4^{+}\right),\left(4^{-}, 5^{+}\right)$: Assume that, for a locally finite connected transitive digraph $D, 4^{-}$ and one of the properties $4^{+}$or $5^{+}$are satisfied. Since $D / R_{-\infty, 0}$ is a chain, we know that $R_{-\infty, 0}=R_{-\infty,+\infty} \supseteq R_{0,+\infty}$. Fix $x \in V(D)$. Since $D$ is locally finite, $S_{+1}(x):=$ $\left\{y \in V(D): x S_{+1} y\right\}$ is a finite subset of the set $R_{0,+\infty}(x) \subseteq R_{-\infty, 0}(x)$. It follows from 
$R_{-\infty, 0}(x)=\cup_{k \geq 0} R_{-k, 0}(x)$ that $S_{+1}(x) \subseteq R_{a, 0}(x)$ for some non-positive integer $a$. Since $D$ is transitive, we conclude that $S_{+1} \subseteq R_{a, 0}$. Now assertion (2) of Proposition 2.3 implies that $R_{0,1} \subseteq R_{a, 0}$. Hence $R_{a, 1} \subseteq R_{a, 0}$ (assertion (2) of 2.1). Assertion (2) of Proposition 2.5 implies that $R_{a, 0}=R_{a,+\infty}$. But then assertion (1) of Corollary 2.4 implies that $R_{0,-a}=R_{0,+\infty}$, a contradiction.

$\left(\mathbf{5}^{-}, \mathbf{5}^{+}\right)$: Any regular directed tree with in-degree $>1$ and out-degree $>1$ has both properties.

\section{Reachability relations in various classes of digraphs}

Considering Theorem 1.1 it is quite natural to investigate the question which pairs of properties $i^{-}, j^{+}$can occur simultaneously in digraphs from important subclasses of the class of locally finite connected transitive digraphs. In this Section we consider the following subclasses: the class of locally finite Cayley digraphs of groups, locally finite connected transitive digraphs with one with two or with infinitely many ends, locally finite connected transitive digraphs containing certain directed subtrees, and locally finite connected highly arc transitive digraphs.

Let $G$ be a group generated by $M \subseteq G$ and $1 \notin M$. Recall that the Cayley digraph of $G$ with respect to $M$ is the digraph $D_{G, M}$ with $V\left(D_{G, M}\right)=G$ and $E\left(D_{G, M}\right)=\left\{(x, y): x^{-1} y \in\right.$ $M\}$. The digraph $D_{G, M}$ is connected and transitive with $\operatorname{deg}^{-}\left(D_{G, M}\right)=\operatorname{deg}^{+}\left(D_{G, M}\right)=$ $|M|$. A digraph $D$ is a Cayley digraph if $D \cong D_{G, M}$ for some group $G$ and some generating set $M$ with $1 \notin M$ of $G$.

To investigate locally finite Cayley digraphs we need the following result.

Proposition 4.1. Let $G$ be a group generated by $M \subseteq G$ and $1 \notin M$. Let $D=D_{G, M}$ be the Cayley digraph of $G$ with respect to $M$. Let $a \in \mathbf{Z}_{\leq \mathbf{0}}$ and $b \in \mathbf{Z}_{\geq \mathbf{0}}$. Then the equivalence classes on $V(D)=G$ with respect to $R_{a, b}$ coincide with the left cosets in $G$ of the subgroup generated by $\cup_{a \leq m \leq b} M^{m} M^{-m}$.

Proof. Let $n$ be an arbitrary positive integer. It is easy to see that, for $g_{1}, g_{2} \in G$, $g_{1} S_{-n} g_{2}$ if and only if there exist $0 \leq m \leq n$ and $x_{1}, \ldots, x_{m} \in M, y_{1}, \ldots, y_{m} \in M$ such that $g_{1}=g_{2} x_{1}^{-1} \ldots x_{m}^{-1} y_{1}, \ldots, y_{m}$. Analogously, for $g_{1}, g_{2} \in G, g_{1} S_{n} g_{2}$ if and only if there exist $0 \leq m \leq n$ and $x_{1}, \ldots, x_{m} \in M, y_{1}, \ldots, y_{m} \in M$ such that $g_{1}=g_{2} x_{1} \ldots x_{m} y_{1}^{-1} \ldots y_{m}^{-1}$. Now Proposition 4.1 follows from Proposition 2.1 and Proposition 2.3.

Theorem 4.2. For locally finite Cayley digraphs the table coincides with the table presented in Theorem 1.1.

Proof. We first show that the $\left(3^{-}, 4^{+}\right)$-entry of the table is $Y$. Let $d$ be a positive integer, and $G:=\left\langle f_{1}, \ldots, f_{d}, h: h^{-1} f_{i} h=f_{i}^{2}, 1 \leq i \leq d\right\rangle$. Then $F:=\left\langle f_{1}, \ldots, f_{d}\right\rangle$ is a free subgroup of $G$ with free generating set $\left\{f_{1}, \ldots, f_{d}\right\}$. Furthermore $h^{-1} F h$ is a subgroup 
of $F$ such that the index $\left|F: h^{-1} F h\right|$ is infinite in the case $d>1$. The index is equal to 2 in the case $d=1$. In addition $U:=\cup_{i \in \mathbf{Z}} h^{-i} F h^{i}$ is a normal subgroup of $G$ such that $G=U \lambda\langle h\rangle$ is a semidirect product of $U$ by the infinite cyclic group $\langle h\rangle$. Let $M:=\left\{h, h f_{1}, \ldots, h f_{d}\right\}$. Then $M$ is a generating set of $G$ and $1 \notin M$. Let $D=D_{G, M}$ be the Cayley digraph of $G$ with respect to $M$. By Proposition 4.1 , for any $a \in \mathbf{Z}_{\leq \mathbf{0}}$ and $b \in \mathbf{Z}_{\geq \mathbf{0}}$, the equivalence classes on $V(D)=G$ with respect to $R_{a, b}$ coincide with the left cosets in $G$ of the subgroup generated by $\cup_{a \leq m \leq b} M^{m} M^{-m}$. But it is easy to see that, in the case $a<0$ or $b>0$, the subgroup generated by $\cup_{a \leq m \leq b} M^{m} M^{-m}$ is $h^{b} F h^{-b}$. Thus, in the case $a<0$ or $b>0$, the equivalence classes on $V(D)=G$ with respect to $R_{a, b}$ coincide with the left cosets in $G$ of the subgroup $h^{b} F h^{-b}$. It follows that the equivalence classes on $V(D)=G$ with respect to $R_{0,+\infty}$ coincide with the left cosets in $G$ of the subgroup $U$, and $R_{0,+\infty} \neq R_{0, b}$ for any $b \in \mathbf{Z}_{\geq \mathbf{0}}$. Furthermore $D / R_{0,+\infty}$ is a chain, since $G / U$ is an infinite cyclic group generated by $h U=M U$. It also follows that the equivalence classes on $V(D)=G$ with respect to $R_{-\infty, 0}$ coincide with the left cosets in $G$ of the subgroup $F$, and, in addition, $R_{-\infty, 0}=R_{-1,0}$. To find the in-degree of the digraph $D / R_{-\infty, 0}$, note that, for $g \in G,(g F, F) \in E\left(D / R_{-\infty, 0}\right)$ if and only if $g \in F h^{-1} F=h^{-1} h F h^{-1} F=h^{-1} h F h^{-1}$. Thus the in-degree of the digraph $D / R_{-\infty, 0}$ is equal to the index $\left|h F h^{-1}: F\right|$ which is infinite in the case $d>1$ and is equal to 2 in the case $d=1$. Thus the Cayley digraph $D$ has properties $3^{-}$and $4^{+}$. Hence the $\left(3^{-}, 4^{+}\right)$-entry of the table is $Y$.

Replacing, in the definition of $G$, the relations $h^{-1} f_{i} h=f_{i}^{2}, 1 \leq i \leq d$, by the relations $h f_{i} h^{-1}=f_{i}^{2}, 1 \leq i \leq d$, and repeating the arguments given above, we get that the $\left(4^{-}, 3^{+}\right)$-entry of the Table is $Y$ as well.

Any (non-trivial) cycle is a Cayley digraph and has properties $1^{-}$and $1^{+}$simultaneously. (Furthermore, any Cayley digraph containing a closed walk of a non-zero height has properties $1^{-}$and $1^{+}$simultaneously, see the proof of Theorem 1.1.) Thus the $\left(1^{-}, 1^{+}\right)$entry of the table is $Y$. Since a chain is a Cayley digraph of $\mathbf{Z}$ and a regular directed tree $T$ with $\operatorname{deg}^{-}(T)=\operatorname{deg}^{+}(T)>1$ is a Cayley digraph of a free group, the entries $\left(2^{-}, 2^{+}\right)$ and $\left(5^{-}, 5^{+}\right)$of the table are $Y$ as well.

Let $D$ be a digraph. An infinite sequence $\left(v_{0}, v_{1}, \ldots\right)$ of pairwise distinct vertices of $D$ is called a ray in $D$ if $\left(v_{i}, v_{i+1}\right) \in E(D)$ or $\left(v_{i+1}, v_{i}\right) \in E(D)$ for each non-negative integer $i$. Two rays $R$ and $Q$ in a digraph $D$ are called equivalent if, in the underlying graph $\bar{D}$, there are infinitely many pairwise disjoint finite paths connecting vertices in $P$ to vertices in $Q$. The equivalence classes of all rays of a digraph $D$ with respect to this relation are called ends of $D$. The concept of ends can be defined in several different ways; this definition is due to Halin [4]. It follows from results in [3], [5] that a transitive, connected, infinite digraph has one, two or infinitely many ends.

Theorem 4.3. (1) For locally finite connected transitive one-ended digraphs, the table is the same as in Theorem 1.1.

(2) For locally finite connected transitive two-ended digraphs, only the entries $\left(1^{-}, 1^{+}\right)$ and $\left(2^{-}, 2^{+}\right)$of the table equal $Y$; all other entries of the table are equal to $N$. 
(3) For locally finite connected transitive digraphs with infinitely many ends, the table differs from the table in Theorem 1.1 only in the entry $\left(2^{-}, 2^{+}\right)$which is $N$ in this case.

Proof. (1) For an arbitrary digraph $D$, define the digraph $D \nearrow \mathbf{Z}$ by $V(D \nearrow \mathbf{Z})=$ $V(D) \times \mathbf{Z}$ and $E(D \nearrow \mathbf{Z})=\{((x, i),(y, i)):(x, y) \in E(D), i \in \mathbf{Z}\} \cup\{((x, i),(y, i+$ 1)) : $(x, y) \in E(D), i \in \mathbf{Z}\}$. It is obvious that, in the case the out-degree of every vertex of $D$ is $\geq 1$ and the in-degree of every vertex of $D$ is $\geq 1$, for each $(x, i) \in$ $V(D \nearrow \mathbf{Z})$ we have $R_{-1,0}^{D \nearrow \mathbf{Z}}((x, i))=\left\{\left(x^{\prime}, i^{\prime}\right): x^{\prime} \in R_{-1,0}^{D}(x), i^{\prime} \in \overline{\mathbf{Z}}\right\}$ and $R_{0,1}^{D \nearrow \mathbf{Z}}((x, i))=$ $\left\{\left(x^{\prime}, i^{\prime}\right): x^{\prime} \in R_{0,1}^{D}(x), i^{\prime} \in \mathbf{Z}\right\}$. In addition, $D \nearrow \mathbf{Z}$ is transitive if $D$ is transitive, and $D \nearrow \mathbf{Z}$ is one-ended if $D$ is infinite and connected. Now assertion (1) of Theorem 4.3 follows from Proposition 2.1 and Theorem 1.1. (Of course, there are infinite locally finite connected transitive digraphs which have properties $1^{-}$and $1^{+}$simultaneously as the proof of Theorem 1.1 in Section 3 shows.)

(2) Assertion (2) of Theorem 4.3 is obvious.

(3) It follows from the proof of Theorem 1.1 (see Section 3), that to prove assertion (3) of the Theorem it is sufficient to show that the $\left(1^{-}, 1^{+}\right)$-entry of the table is $Y$, while the $\left(2^{-}, 2^{+}\right)$-entry of the table is $N$.

Any locally finite connected transitive digraphs containing a closed walk of a non-zero height has properties $1^{-}$and $1^{+}$simultaneously (see the proof of Theorem 1.1 in Section 3). Since there exist locally finite connected transitive infinitely-ended Cayley digraphs containing closed walks of non-zero height (take, for example, the Cayley digraph of the free product $\left\langle g_{1}\right\rangle *\left\langle g_{2}\right\rangle$ of cyclic groups of orders $>2$ with respect to the generating set $\left.\left\{g_{1}, g_{2}\right\}\right)$, the $\left(1^{-}, 1^{+}\right)$-entry of the table is $Y$.

If a transitive digraph $D$ has both properties, $2^{-}$and $2^{+}$, simultaneously, then $D$ has property $\mathbf{Z}$ (see Corollary 2.12). But as was shown in [8], any digraph with infinitely many ends and property $\mathbf{Z}$ has the property that at least one of the conditions $R_{-\infty, 0} \neq R_{a, 0}$ for all negative integers $a$, or $R_{0,+\infty} \neq R_{0, b}$ for all positive integers $b$ is satisfied. Hence $2^{-}$and $2^{+}$cannot occur simultaneously.

To formulate our next result we define: A digraph $D$ is of type $\mathrm{T}^{-}$if it contains a regular directed tree with in-degree $>1$ but does not contain a regular directed tree with out-degree $>1$; it is of type $\mathrm{T}^{+}$if it contains a regular directed tree with out-degree $>1$ but does not contain a regular directed tree with in-degree $>1$. D is of type $\mathrm{T}^{ \pm}$if it contains a regular directed tree with in-degree $>1$ as well as a regular directed tree with out-degree $>1$. It is of type NT if it neither contains a regular directed tree with in-degree $>1$ nor a regular directed tree with out-degree $>1$.

Theorem 4.4. Let $D$ be a connected locally finite transitive digraph. The table whose $\left(i^{-}, j^{+}\right)$-entry is $Y$ if $D$ can have properties $i^{-}$and $j^{+}$simultaneously, and is $N$ otherwise, is as follows:

(1) for $D$ of type $\mathrm{T}^{ \pm}$, the table coincides with the table of Theorem 1.1. 
(2) for $D$ of type $\mathrm{T}^{-}$, the table differs from the table of Theorem 1.1 only in the entries $\left(4^{-}, 3^{+}\right)$and $\left(5^{-}, 5^{+}\right)$which are $N$ in this case.

(3) for $D$ of type $\mathrm{T}^{+}$, the table differs from the table of Theorem 1.1 only in the entries $\left(3^{-}, 4^{+}\right)$and $\left(5^{-}, 5^{+}\right)$which are $N$ in this case.

(4) for $D$ of type NT, the table differs from the table of Theorem 1.1 only in the entries $\left(3^{-}, 4^{+}\right),\left(4^{-}, 3^{+}\right)$and $\left(5^{-}, 5^{+}\right)$which are $N$ in this case.

Proof. Obviously, any cycle is of type $N T$ and has properties $1^{-}$and $1^{+}$. Let $T_{m, n}$ denote the regular directed tree with in-degree $m$ and out-degree $n$, with $m, n \geq 1$. Let $T_{m, n}^{2}$ denote the digraph with $V\left(T_{m, n}^{2}\right)=V\left(T_{m, n}\right)$ and $E\left(T_{m, n}^{2}\right)=E\left(T_{m, n}\right) \cup\{(u, v)$ : there exists $w \in V\left(T_{m, n}\right)$ such that $(u, w) \in E\left(T_{m, n}\right)$ and $\left.(w, v) \in E\left(T_{m, n}\right)\right\}$. Put $D_{1}=T_{2,2}^{2}$ and $D_{2}=T_{2,1}^{2}$. By Proposition 2.9 and Corollary 2.8, each of digraphs $D_{1}, D_{2}$ has properties $1^{-}$and $1^{+}$simultaneously. Furthermore, it is easy to see that $D_{1}$ is of type $\mathrm{T}^{ \pm}$while $D_{2}$ is of the type $\mathrm{T}^{-}$and $D_{2}^{*}$ is of the type $\mathrm{T}^{+}$. Finally, any cycle is of type NT and has properties $1^{-}$and $1^{+}$. Thus the $\left(1^{+}, 1^{-}\right)$-entries in the tables for all types $\mathrm{T}^{ \pm}, \mathrm{T}^{+}, \mathrm{T}^{-}$and NT are $Y$.

For an arbitrary digraph $D$, define the digraph $D \uparrow \mathbf{Z}$ by $V(D \uparrow \mathbf{Z})=V(D) \times \mathbf{Z}$ and $E(D \uparrow \mathbf{Z})=\{((x, i),(x, i+1)): x \in V(D), i \in \mathbf{Z}\} \cup\{((x, i),(y, i+1)):(x, y) \in$ $E(D), i \in \mathbf{Z}\}$. If $D$ is connected it is obvious that for every $(x, i) \in V(D \uparrow \mathbf{Z})$ we get $R_{-1,0}^{D \uparrow \mathbf{Z}}((x, i))=R_{0,1}^{D \uparrow \mathbf{Z}}((x, i))=\left\{\left(x^{\prime}, i\right): x^{\prime} \in V(D)\right\}$. In addition $D \uparrow \mathbf{Z}$ is transitive if $D$ is transitive. Furthermore, if $D$ contains a regular directed tree $T$ of in-degree $>1$ (respectively, a regular directed tree $T^{*}$ of in-degree $>1$ ), then $D \uparrow \mathbf{Z}$ contains $T \uparrow \mathbf{Z}$ (respectively, $T^{*} \uparrow \mathbf{Z}$ ) which contains a regular directed tree of in-degree (respectively, of out-degree) $>1$. It follows, the digraphs $T_{2,2} \uparrow \mathbf{Z}, T_{2,1} \uparrow \mathbf{Z}$ and $T_{1,2} \uparrow \mathbf{Z}$ are of types $\mathrm{T}^{ \pm}$, $\mathrm{T}^{-}$and $\mathrm{T}^{+}$, respectively, and have properties $2^{+}$and $2^{-}$simultaneously. Finally, a chain is of type NT and has properties $2^{-}$and $2^{+}$. Thus the $\left(2^{-}, 2^{+}\right)$-entries in the tables for all types $\mathrm{T}^{ \pm}, \mathrm{T}^{+}, \mathrm{T}^{-}$and $\mathrm{NT}$ are $Y$.

To complete the proof of assertion (1) we note that any regular directed tree of indegree $>1$ and of out-degree $>1$ is of type $\mathrm{T}^{ \pm}$and has properties $5^{-}$and $5^{+}$simultaneously. Furthermore, let $D_{G, M}$ be the Cayley digraph defined in the proof of Theorem 4.2. It is easy to see that the subsemigroup of $G$ generated by the subset $\left\{h f_{1}, \ldots, h f_{d}\right\}$ of the set $M$ is free. Hence the subset $\left\{\left(h f_{1}\right)^{-1}, \ldots,\left(h f_{d}\right)^{-1}\right\}$ of the set $M^{-1}$ also generates a free subsemigroup. In the case $d>1$, it follows that the digraph $D_{G, M}$ is of type $\mathrm{T}^{ \pm}$. Since $D_{G, M}$ has properties $3^{-}$and $4^{+}$(see the proof of Theorem 4.2 ), the $\left(3^{-}, 4^{+}\right)$-entry of the table corresponding to the type $\mathrm{T}^{ \pm}$is $Y$. Furthermore, in the case $d>1$, the digraph $D_{G, M}^{*}$ is of type $\mathrm{T}^{ \pm}$and has properties $4^{-}$and $3^{+}$. Thus the $\left(4^{-}, 3^{+}\right)$-entry of the table corresponding to the type $\mathrm{T}^{ \pm}$is $Y$ as well. The proof of the assertion (1) of Theorem 4.4 is completed.

To complete the proof of assertion (2) of Theorem 4.4, note that any regular directed tree with in-degree $>1$ and out-degree 1 is of type $\mathrm{T}^{-}$and has properties $3^{-}$and $4^{+}$. Furthermore, the assertion (2) of Proposition 2.14 implies that every connected transitive digraph satisfying property $4^{-}$or property $5^{-}$contains a regular tree with in-degree 1 and out-degree 2. In particular, digraphs of type $\mathrm{T}^{-}$neither have property $4^{-}$nor property 
$5^{-}$. Thus assertion (2) holds.

Assertion (3) of Theorem 4.3 can be proved analogously (using a regular directed tree with in-degree 1 and out-degree $>1$ to show that the $\left(3^{-}, 4^{+}\right)$-entry of the corresponding table is $Y$, and using assertion (1) of Proposition 2.14 to show that $\left(4^{-}, 3^{+}\right)-,\left(5^{-}, 5^{+}\right)$entries of the corresponding table are $N$ ).

Assertion (4) of Theorem 4.4 follows from Proposition 2.13.

Remark. Following [11] we say that a digraph $D$ is in-hyperbolic with respect to $v$, where $v \in V(D)$, if there exists a positive integer $t$ such that the digraph $D^{t}$ (see Section 2) contains a directed tree $T$ with the following properties: $v \in V(T)$ and the out-degree of $v$ is 0 ; the out-degree of any vertex of $T$, different from $v$, is 1 ; the in-degree of any vertex of $T$ is 2. A digraph $D$ is out-hyperbolic with respect to $v$, where $v$ is a vertex of $D$, if the digraph $D^{*}$ is in-hyperbolic with respect to $v$. If a digraph $D$ is transitive and in-hyperbolic (respectively, transitive and out-hyperbolic) with respect to some vertex, then $D$ is in-hyperbolic (respectively, out-hyperbolic) with respect to any vertex. We will call such graphs in-hyperbolic (respectively, out-hyperbolic).

Analogously to the above we can define the following classes of locally finite connected transitive digraphs $D$ :

(1) $D$ is in-hyperbolic and out-hyperbolic;

(2) $D$ is in-hyperbolic but not out-hyperbolic;

(3) $D$ is not in-hyperbolic but out-hyperbolic;

(4) $D$ is neither in-hyperbolic nor out-hyperbolic.

An analysis of the proof of Theorem 4.4 shows that, for digraphs $D$ satisfying (1), (2), (3) or (4), the table whose $\left(i^{-}, j^{+}\right)$-entry is $Y$ if $D$ can have properties $i^{-}$and $j^{+}$ simultaneously, and is $N$ otherwise, is given, respectively, by the assertion (1), (2), (3) or (4) of Theorem 4.4.

An $s$-arc, $s \geq 0$, is a walk $\left(v_{0}, e_{1}^{\epsilon_{1}}, v_{1}, \ldots, v_{s-1}, e_{s}^{\epsilon_{s}}, v_{s}\right)$ such that $\epsilon_{1}=\ldots=\epsilon_{n}=1$ and $v_{j} \neq v_{j+2}$ for all $0 \leq j \leq s-2$. If $\operatorname{Aut}(D)$ acts transitively on the set of $s$-arcs, then $D$ is called s-arc transitive. $D$ is said to be highly arc transitive if it is $s$-arc transitive for all integers $s \geq 0$. Highly arc transitive digraphs are an interesting class of digraphs which were first intensively investigated in [1]. For further investigations and applications of highly arc transitive digraphs we refer to [2], [6], [7], [9], [10].

Theorem 4.5. For connected infinite locally finite connected highly arc transitive digraphs the table coincides with the table in Theorem 1.1.

Proof. Obviously the examples of digraphs satisfying $\left(2^{-}, 2^{+}\right),\left(3^{-}, 4^{+}\right),\left(3^{+}, 4^{-}\right)$and $\left(5^{-}, 5^{+}\right)$given in the proof of Theorem 1.1 are all highly arc transitive. As was shown in [6], the digraph below (Figure 1) 


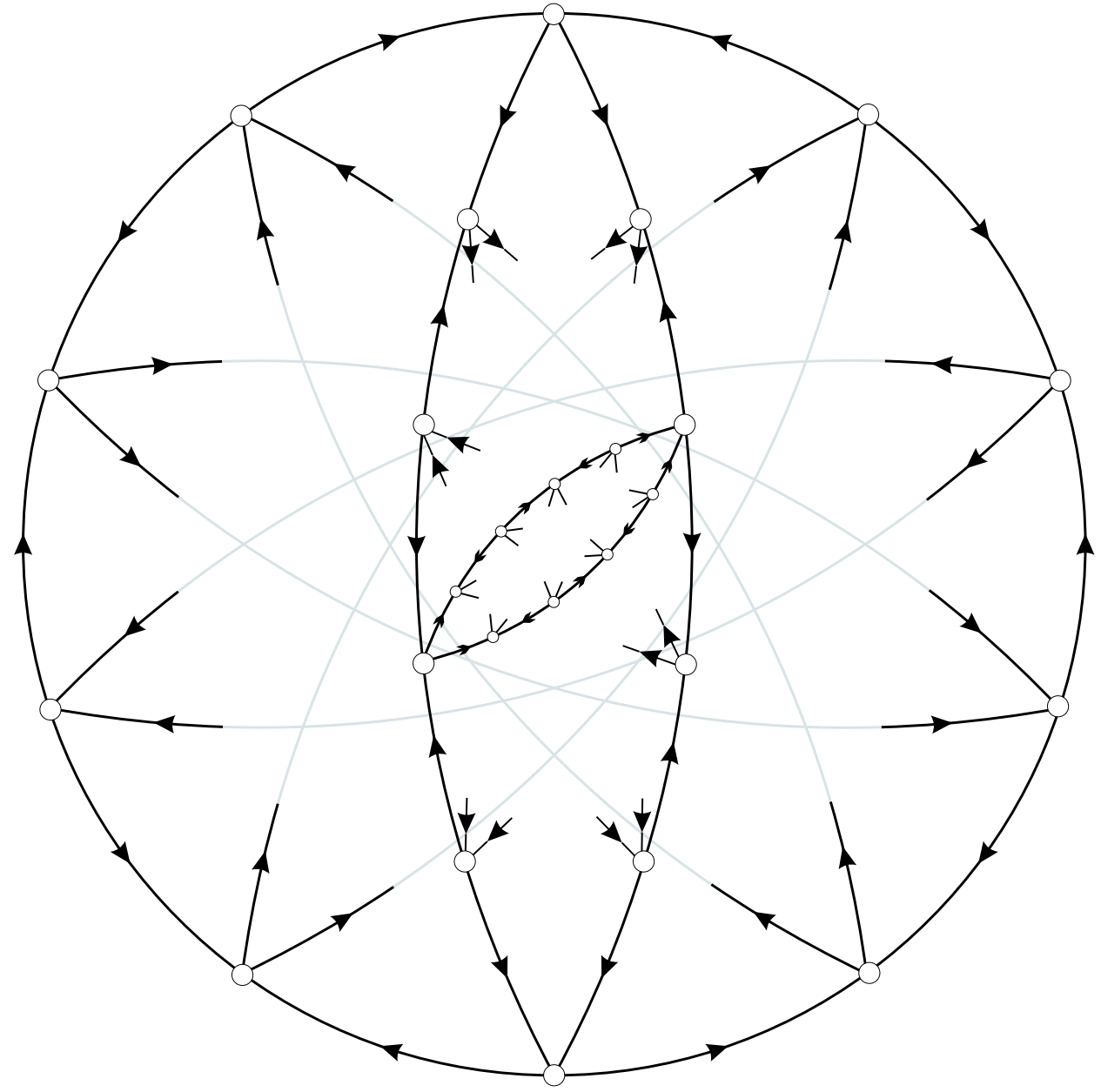

Figure 1

is highly arc transitive and does not have property $\mathbf{Z}$. Hence $R_{0,+\infty}=R_{0, b}$ and $R_{-\infty, 0}=$ $R_{a, 0}$ for some $b \in \mathbf{Z}_{\geq 0} \cup\{+\infty\}, a \in \mathbf{Z}_{\leq 0} \cup\{-\infty\}$ (see Proposition 2.10). Also, the quotient digraphs $D / R_{0,+\infty}$ and $D / R_{-\infty, 0}$ cannot be infinite since this would again imply that $D$ has property $\mathbf{Z}$ (see Corollary 2.12). Hence this graph satisfies $1^{-}$as well as $1^{+}$. The result follows.

\section{References}

[1] P. J. Cameron, C. E. Praeger, N. C. Wormald, Infinite highly arc transitive digraphs and universal covering digraphs, Combinatorica 13 (1993), 377-396.

[2] D. M. Evans, An infinite highly arc-transitive digraph, European J. Combin. 18 (1997), $281-286$. 
[3] R. Halin, Automorphisms and endomorphisms of infinite locally finite graphs, Abh. Math. Sem. Univ. Hamburg 39 (1973), 251 - 283.

[4] R. Halin, Über unendliche Wege in Graphen, Math. Ann. 157 (1964), 125 - 137.

[5] H. A. Jung, A note on fragments of infinite graphs, Combinatorica 1 (1981), 285 288.

[6] A. Malnič, D. Marušič, N. Seifter, B. Zgrablič, Highly arc-transitive digraphs with no homomorphism onto Z, Combinatorica 22 (2002), 435 - 443.

[7] A. Malnič, D. Marušič, R. Möller, N. Seifter, V. Trofimov, B. Zgrablič, Highly arc transitive digraphs: reachability, topological groups, Europ. J. Combinatorics 26 (2005), $19-28$.

[8] A. Malnič, D. Marušič, N. Seifter, P. Sparl, B. Zgrablič, Reachability relations in digraphs, Europ. J. Combinatorics 29 (2008), 1566-1581.

[9] R. G. Möller, Descendants in highly arc-transitive digraphs, Disc. Math. 247 (2002), $147-157$.

[10] R. G. Möller, Structure theory of totally disconnected locally finite groups via graphs and permutations, Canad. J. Math. 54 (2002), 795 - 827.

[11] V. I. Trofimov, Undirected and directed graphs with near polynomial growth, Discuss. Math. Graph Theory 23 (2003), 383 - 391.

[12] V. I. Trofimov, Growth functions of permutation groups, Trudy Inst. Mat. (Proc. of the Inst. of Math.), 4, Nauka, Novosibirsk, (1984), 118 - 138. (Russian) 